


\section{EDUCACIÓN AMBIENTAL: SABER INTERDISCIPLINAR Y TRANVERSAL*}

\section{Environmental Education, Interdisciplinary and transversal knowledge}

GUSTAVO ADOLFO BONILLA PÉREZ ${ }^{1}$

\section{Resumen}

La educación ambiental es un proceso que propende por involucrar a los seres humanos dentro de la reflexión del cuidado ambiental, garantizando la armonía entre los individuos y su esfera natural, como engranajes de la biosfera, en el que se desarrollan interacciones de cooperativismo en pro de una sustentabilidad de los recursos.

Para garantizar que la educación alcance ese propósito esencial, se requiere de la introducción formativa de la dimensión ambiental en su integralidad socio-cultural. Esta realidad implica un tratamiento de la problemática ambiental de manera coherente y significativa, que propicie que la actividad cognoscitiva de los alumnos se encuentre en constante desarrollo para integrar conocimientos. (Covas Álvarez, 2004, p. 1).

La naturaleza, gran tesoro de la humanidad que aunque colosal e invaluable, no es ilimitada. Para lo anterior hemos de tener como referente, tanto el desarrollo de habilidades del pensamiento innovador y transformador en el ser social, como el desarrollo de un comportamiento ambiental. El hecho es que no hemos sido capaces de provocar un cambio hacia una actitud positiva-crítica-ambiental frente a este tipo de problema. Se nos convierte entonces como un objetivo primordial enseñar a aprender con la intencionalidad de conocer, comprender, actuar y provocar cambios efectivos en nuestro deteriorado entorno natural. En este sentido, lo que enseñamos y cómo lo enseñamos es parte del problema. Para esto, pensamos que es necesario propender por un conocimiento significativo; si lo tomamos claro está, como aquel aprendizaje que tiene sentido; integrando los nuevos elementos con los que anteriormente habíamos asimilado y sabiendo utilizarlo de forma creadora, transformadora, útil y práctica para el bienestar principalmente, de la naturaleza $y$, por ende, para nuestra vida y permanencia en el mundo.

Palabras clave: educación ambiental, cultura, problemas ambientales, conciencia, interdisciplinariedad.
Fecha de recepción: 29 de abril de 2013 Fecha de aprobación: 10 de mayo de 2013

\section{Abstract}

Environmental education is a process which tends to involve human beings within the reflection of environmental care, ensuring harmony between people and their natural sphere, as gears in the biosphere, in which interactions of cooperativism on behalf resource sustainability are developed.

To ensure that education reaches that essential purpose, the introduction of the environmental dimension training in its socio-cultural integrity is required. This reality involves a treatment of environmental issues in a coherent and meaningful way, which contributes to students' cognitive activity that is in constant development to integrate knowledge. (CovasÁlvarez, 2004, p. 1).

The nature, great treasure of humanity that although it is colossal and invaluable. It is not unlimited. To the above we take into account both the development of innovative and transformative thinking skills in social beings, as the development of an environmental behavior. The fact is that we have not been able to arouse a change towards a positive and critical environmental attitude- facing this kind of problem. For this a primary goal was to teach to learn with the intention of knowing, understanding acting and provoking effective changes in our deteriorated natural environment. In this sense, what we teach and how we teach is part of the problem. For this, we think it is necessary to tend to a meaningful knowledge, if we take it, of course, as that learning that makes sense, integrating new elements that were previously assimilated and using them in a creative, transformative, useful and practical way for mainly the welfare of nature and thus our life and our stay in the world.

Keywords: Environmental Education, Culture, Environmental Issues, Consciousness, Interdisciplinary.

* El artículo se deriva de un trabajo de un estudio y reflexión, en el quehacer docente durante tres años en el Instituto San Carlos de la Salle, al interior del Área de Ciencias Naturales y Educación ambiental.

1 Licenciado en Educación Básica con Énfasis en Ciencias Naturales y Educación Ambiental de la Facultad de Educación de la Universidad de Antioquia. Docente Instituto San Carlos de la Salle. Área de Ciencias Naturales y Educación Ambiental.

Correo electrónico: gustavo.bonilla@delasalle.edu.co, tavobon@yahoo.com 
La educación ambiental no puede y no debe verse simplemente como una asignatura más, como una ciencia aislada, es un conjunto de saberes y conocimientos que parten de la interacción del sujeto con su medio. En palabras de Leff (2002):

El saber ambiental, emerge de una reflexión, sobre la construcción social del mundo actual, tiempo donde surgen nuevos valores y racionalidades, que reconducen su construcción. El saber ambiental, crítico y complejo, se va construyendo en un diálogo de saberes y un cambio interdisciplinario de conocimientos; va constituyendo un campo epistémico, que problematiza los paradigmas establecidos, para construir una nueva racionalidad social (p. 211).

Se sabe que la educación se ha alejado demasiado de su verdadero objetivo como ente formador de ciudadanos autónomos e integrales, capaces de vivir en armonía con su entorno natural. Hoy solo se preocupa por enseñar conceptos y un sinnúmero de teorías que en nada ayudan a la toma de una conciencia y una acción ambiental de equilibrio, que beneficie en igual medida a ambas esferas: la social y la natural. Por otro lado, la educación es la madre de todo conocimiento y este es el responsable de la formación de una cultura.

\begin{abstract}
[...] escuela en cuanto sistema social y democrático, debe educar para que los individuos y las colectividades comprendan la naturaleza compleja del ambiente, resultante de la interacción de sus aspectos biológicos, físicos, químicos, sociales, económicos y culturales; construyan valores y actitudes positivas para el mejoramiento de las interacciones hombre-sociedad-naturaleza, para un manejo adecuado de los recursos naturales y para que desarrollen las competencias básicas para resolver problemas ambientales. (Ministerio de Educación Nacional, 1998).
\end{abstract}

De un modo de acción dentro del mundo biofísico se ha de formar, entonces, un ser humano integral y culturalmente capaz de vivir en armonía con su medio social y principalmente con su medio natural. Si los fines de la educación van hacia una vida en equilibrio, entonces, ¿por qué no se está formando ciudadanos de acción que posean la capacidad necesaria de contextualizar sus conocimientos teóricos y utilizarlos en el mundo real?

¿Por qué la educación ambiental se da como una asignatura más, teniendo muy claro que es un conocimiento interdisciplinario y transversal? De acuerdo con la ONU, la educación ambiental tiene como objetivo formar en los estudiantes una conciencia de los problemas de su entorno natural en la vida cotidiana, proporcionando conocimientos, capacidades y responsabilidades indispensables para encontrar solución a dichos problemas. Por otro lado, un argumento para consolidar la educación ambiental como eje transversal es el establecido por la Secretaría de Ambiente y el Ministerio de Educación de Argentina en 2009:

Ambiente hace referencia al enlace entre lo material y lo simbólico, al reconocimiento y apropiación del mundo natural y social. Ambiente, entendido como fuente de recursos para el desarrollo del ser humano y como ámbito cultural donde pueda comprenderse la historia, el progreso y la proyección futura. La educación ambiental se incluye como contenido transversal a partir de un posicionamiento de abordaje curricular integrador de las diferentes disciplinas que permitan un análisis crítico del propio ambiente en su globalidad y complejidad. (Pág. 3)

Si el hombre tiene conocimiento de su fuerte y negativa influencia en el ambiente, ¿por qué no hace nada para garantizar su supervivencia y su calidad de vida?, ¿acaso es un problema de unos cuantos y no de todos? Pareciera que los problemas ambientales tuvieran su mejor (única) solución en la esfera de la técnica y las ciencias naturales, y no en las transformaciones sociales y los cambios de comportamiento -cultura- de los individuos. Sabemos que por definición, los problemas ambientales exigen un tratamiento interdisciplinario y transversal.

En cuanto a lo anterior, muchos estudios actuales en torno a la realidad holística del hombre, plantean la necesidad de una integración mayor entre lo objetivo y lo subjetivo, entre lo comportamental y lo cognoscitivo, entre lo experimental y lo social, esto es, entre las disciplinas que apuntan a lo natural y lo social.

Para ello necesita un maestro con un alto nivel de integridad, que garantice además de los conocimientos necesarios, el desarrollo de habilidades y la formación de valores que hoy exige nuestra sociedad para el cuidado y conservación de nuestro entorno, un maestro que eduque ambientalmente a nuestros estudiantes. (Covas Álvarez, 2004, p. 6).

De ahí la necesidad de incluir en el currículo la dimensión ambiental con una perspectiva holística, desarrolladora, tal como se establece en los principios de la educación ambiental hacia sociedades sustentables. El fin de integrar conceptos a nuestra realidad social es el reto de la moderna visión de la investigación, para brindar una solución acorde con la problemática social y ambiental actual, entendiendo que, sin embargo, se pretende ligar al individuo y su comportamiento grupal y colectivo con su medio natural y social, como un contexto de interacción que busca el equilibrio.

\section{[...] Por tanto, se identifica la educación ambiental como un problema, ya que encontrándose sobre la plataforma del deber ser, se observa y se entiende como una materia más con contenidos específicos al interior del currículo y no como un nuevo enfoque holístico dentro del quehacer educativo. (Mahecha, 2003, p. 89).}

De acuerdo con lo anterior, al parecer la educación solo está direccionada hacia el aspecto teórico y limitada por un aula, en la cual se dan los conocimientos, pero sin las herramientas y sin la posibilidad de llevarlos a la aplicación en un contexto real, como lo es nuestra propia vida, nuestra sociedad. Solamente se está enseñando, mas no formando; proceso que implica la toma de conciencia, reflexión, análisis; pero con la diferencia de que las decisiones trascienden, se contextualizan y toman cuerpo en la acción, haciéndonos partícipes en nuestra formación académica.

La educación ambiental debería tomar en cuenta el medio natural y artificial en su totalidad: ecológico, político, tecnológico, social, legislativo, cultural y estético; debe- 
ría ser un proceso continuo y permanente en la escuela y fuera de ella; debería tener un enfoque interdisciplinario; debería hacer hincapié en una participación activa en la prevención y solución de los problemas ambientales desde un punto de vista mundial, teniendo en cuenta las diferencias regionales; debería concentrarse en cuestiones ambientales actuales y futuras; debería considerar desarrollo y crecimiento en una perspectiva ambiental; la educación debería fomentar el valor y la necesidad de la cooperación local, nacional e internacional en la resolución de los problemas ambientales (2009. p. 4). (Rengifo, et al. 2012, pág. 4).

La educación es un conglomerado de ciencias, de esferas; es globalidad, puesto que ella está sumergida en una sociedad y un contexto cultural, económico, político, los cuales influyen de manera directa en los objetivos que ha de tener. De acuerdo con Leff (2002), "el ambiente emerge como un saber reintegrador de la diversidad, de nuevos valores éticos y estéticos, de los potenciales sinergéticos que genera la articulación de procesos ecológicos, tecnológicos y culturales". (p. 19)

La educación ambiental es una interacción entre conocimientos y entre el ser humano y su entorno natural; por ese motivo no solo corresponde al interior de un aula sino que, como proceso, también debe incluir el contexto real-cotidiano en el cual se destaca una formación integral y activa para la participación en las soluciones de tipo ambiental, que tienen sus inicios en los comportamientos sociales y culturales.

"No es posible definir las finalidades de la educación ambiental sin tener en cuenta las realidades económicas, sociales y ecológicas de cada sociedad y los objetivos que esta se haya fijado para su desarrollo" (Novo, 1996, p. 81).

La educación ambiental si desea participar y aportar soluciones concretas y reales activamente, tiene la obligación de partir de nuestra realidad: sus objetivos y sus finalidades deben estar contextualizadas, es decir, debe tomar como eje fundamental, la vida social, la realidad.

El objetivo de este trabajo es relacionar la educación ambiental que se desarrolla en ámbitos escolares (educación formal), con aquella otra que vienen realizando las organizaciones no gubernamentales, grupos ecologistas y comunidades autónomas (educación no formal), por considerar que ambas forman parte de un mismo sistema de pensamiento y acción, en el que los avances de una influyen y realimentan los avances de la otra. (Novo, 1996, p. 75).

Una educación ambiental con finalidades concretas y resultados positivos, es aquella que en una primera etapa facilita el acercamiento a los conocimientos; luego se fusiona con la educación no formal para tomar fuerza y revitalizar esos saberes, para que finalmente formen parte activa de un pensamiento divergente con nuevas, novedosas e innovadoras propuestas que contribuyan a un cambio significativo en cuanto a la problemática ambiental,

Entendiendo que la explicación de la problemática ambiental se sitúa no en los síntomas, sino en el centro mis- mo de la actividad humana extractiva o productiva contextualizada por una forma cultural. Es decir en el interior de la cultura y de los procesos de la cultura y de los procesos sociales: económicos, tecnológicos, simbólicos, etc. (González, 1999, p. 32).

La educación ambiental como eje de formación para las personas de una cultura, permite contextualizar y llevar por los caminos conscientes en el pensar, actuar y solucionar la situación de los problemas ambientales en cuanto al modo de educar ambientalmente.

Crear una actitud pedagógica que aborde la enseñanza y el aprendizaje para el desarrollo de la educación ambiental, desde un programa dinámico que epistemológicamente le proporcione vida a los conceptos que se presentan estáticos o muertos en los textos de estudio o en el discurso mismo del maestro. (Mahecha, 2003, p. 89).

Es deseable que la práctica de la educación ambiental no sea un programa más de simple acumulación de conceptos y teorías, en donde predomina la pasividad sino que su eje principal y campo de acción sean la realidad, que los conocimientos aprendidos tomen vida y sean puestos en práctica, predominando la integración de conceptos, actitudes, valores; en una interdisciplinariedad. ¿Qué mejor lugar para la enseñanza de lo ambiental que el mismo ambiente?

Como se observa, la educación es el principio de todo conocimiento de tipo conceptual y actitudinal, ambos son la representación del ser y el deber ser dentro de la sociedad y en la cual se ha de manifestar la formación de un ciudadano íntegro, capaz de convivir en armonía y en equilibrio con los demás seres del medio natural que constituyen su entorno. Por consiguiente, la cultura tiene como principio y como cuna la educación, la cual debe propender por su formación, tendiente a buscar una interacción de equilibrio entre el mundo humano y el mundo natural.

La educación ambiental influye directamente sobre la cultura, por ende en la formación del individuo se deben tocar diferentes esferas: social, educativa, cultural y política; como un conjunto de saberes, valores y actitudes que interactúan y se complementan entre sí, dando origen a las relaciones (sociedad-hombre-naturaleza) con el único fin de formar mujeres y hombres desde la acción, la conciencia y el conocimiento ambiental; además de estar permeados por una ética y una moral de equilibrio ecosistémico, permitiendo contar con una cultura que les permita tomar decisiones en favor del ambiente.

No basta con enseñar desde la naturaleza utilizándola como recurso educativo, hay que educar para el medio ambiente, hay que presentar y aprender conductas correctas hacia el entorno, no solo conocerlo. Se trata de un nuevo entendimiento de las relaciones del ser humano con el entorno: la concepción de la naturaleza no como una fuente inagotable de recursos a nuestro servicio sino como un ecosistema frágil que tiene sus propias exigencias que hay que respetar en nuestro propio interés. Se pasa así de objetivos psicológicos y didácticos a criterios de tipo ecológico. (González 1999. p. 4) 
La tendencia ambientalista que emerge de este escenario concibe al ambiental como un fenómeno cultural, producto de la compleja interacción del hombre con la naturaleza y de las relaciones sociales que emanan de esta interacción, las cuales determinan las condiciones materiales y sociales en las que se reproduce una sociedad. (Ibarra, 1999, p. 72).

El tema ambiental no es un sistema aislado y desarticulado de cualquier otra esfera global, esta se sumerge en las esferas sociales, culturales y económicas conformando una interacción y una transversalidad.

De ahí que la tendencia ambientalista le asigne a la educación una función primordial y pugne por desarrollar un tipo de educación ambiental tendiente a la formación de ciudadanos, cuya participación por la conservación del ambiente se sustente en el conocimiento de los problemas ambientales, culturales, sociales y en una toma de conciencia crítica y responsable (Ibarra, 1999, p. 73).

Una de las finalidades primordiales de la educación, es hacer de la educación ambiental una cultura, pero supeditada a la participación activa y real dentro de los numerosos y diversos problemas que presenta, dirigidos a incentivar el espíritu investigador, reflexivo, crítico y propositivo.

"Esta tendencia conceptualiza al ambiente como totalidad, la cual incluye aspectos naturales, sociales, económicos, políticos, culturales, tecnológicos, éticos y estéticos" (Ibarra, 1999, p. 74). El tema ambiental es global, en el cual se encuentra sumergido el hombre con su complejo existir-vivir. No podemos ni debemos en ningún caso, separar o aislar el ambiente de los demás aspectos que influyen en ella; pues, todo conforma una unidad, un todo.

Como Martin Luther King dijo, en un contexto algo diferente: "Si usted no es parte de la solución, usted es parte del problema".

Los problemas ambientales son lo son también sociales, y son causados por las prácticas educativas que en nada contextualizan sus saberes y las prácticas culturales que parecen no partir de una actitud hacia el cuidado de su entorno natural, que se encuentran enmarcados e influenciados por sus valores.

La educación de la ciencia para la acción sociopolítica es ineludiblemente un ejercicio en la clarificación y cambio de valores medioambientales; los problemas no se marcharán por sí solos, ni se resolverán por un rápido apuro técnico; mientras nosotros mantengamos nuestro estilo de vida libertino, sin que nos importe el mañana. Debemos cambiar la manera de pensar, de actuar, para que a partir de este momento transformemos nuestra forma de vivir y de actuar frente al mundo natural y social, puesto que el planeta ya no puede sostener nuestro presente estilo de vida.

Ya en relación con la parte educativa, se hace necesario implementar a los fundamentos teóricos-conceptuales, el trabajo de campo. Se enseña mucho, pero no se enseña cómo articular los conocimientos a la solución de problemas concretos inscritos dentro en la realidad ambiental. Por esto, hoy la teoría toma significado cuando es puesta en prácti- ca en el espacio escolar (cuidar y proteger las zonas verdes, sembrar árboles y plantas, campañas de concientización en contra del consumismo incontrolado, manejo desechos orgánicos e inorgánicos, prevención desde nuestros hogares, etc.), para los primeros grados. Para los grados superiores se propone como campo de acción su comunidad, su barrio; en donde analicen, propongan y desarrollen estrategias de solución a los problemas ambientales que presenta su entorno (quemas indeliberadas, destrucción de los hábitats naturales, protección de riachuelos o quebradas, tratamiento de basuras, propuestas críticas frente a la actualidad en cuanto a biocombustibles, emisiones de dióxido de carbono, calentamiento global, etc.); son tantas las cosas que se pueden hacer, que es inaudito solo pensar que nos quedamos con el simple hecho de saber teoría pero sin participar, actuar y cambiar nuestro comportamiento y nuestra cultura de deterioro y destrucción, por una cultura de equidad, equilibrio, armonía y felicidad, con nuestro cada vez más agotado mundo natural.

\section{Conclusiones}

No se puede esperar que todo este proceso cambie de la noche a la mañana, ya que transformar imaginarios de una cultura es sumamente complejo y tarda bastante, pero hay que comenzar. Lo importante es que es un movimiento mundial el que está en mejora del medio, unos desde una parte más ecológica y científica, otros desde una parte más social, a nosotros como maestros en formación nos corresponde cuestionar la formación de esa cultura e indagar sobre los resultados en esta para poder brindar soluciones no pasivas ni meramente teóricas, sino que conlleven a propiciar actitudes en mejora del ambiente.

La cuestión ambiental ya no es sólo un referente de estudio para los grupos profesionales especializados, sino que ha entrado de lleno en la agenda de las preocupaciones sociales e, incluso, personales.

El problema que aún sigue abierto, consiste no tanto en definir y describir la preocupación ambiental, sino, sobre todo, de explicar los distintos niveles de conciencia ambiental en función de variables sociales, psicológicas y de visiones del mundo. (Corraliza, 2001. P. 178).

Por consiguiente, es prudente reflexionar sobre el desarrollo de una ética ambiental, que debería llevarse a cabo en la educación ambiental, pues la educación emerge como una de las soluciones más practicable, viable, deseable y oportuna, si se trata de pensar en cambios de actitud del hombre hacia su medio natural, en la modificación de sus concepciones sobre ella y, por supuesto, en la generación de nuevos y adecuados comportamientos, todos ellos unidos hacia un único y claro fin: superar el deterioro acelerado que está presentando nuestro ambiente natural. Se debe educar al hombre desde dos perspectivas básicamente; una es de cara a su realidad, a su entorno natural, con el objetivo de forjar en él su cuidado y preservación, a partir de la sensibilización y acción, con respecto a esos recursos naturales, que 
aunque renovables no son inagotables, puesto que tiene la posibilidad como eje fundamental de una sociedad, de una cultura, propender por aminorar o desaparecer por completo, el gran impacto negativo, acelerado y destructor que él mismo a establecido sobre los recursos naturales, buscando así una interacción de beneficio mutuo, de equilibrio. La otra perspectiva tiene que ver con la educación, espacio de formación hacia la interacción, entre lo social, lo cultural y lo ambiental desde lo sistémico e interdependencia para la sustentabilidad de los recursos y de la misma vida.

La tradición cultural debería ser concebida, de manera reflexiva y crítica, con el fin de que logremos comprender nuestro papel en el ecosistema y poder actuar acorde a las situaciones actuales, mas no por simple inercia y herencia. Somos los únicos responsables de los graves cambios que está presentando nuestro presente natural, pero al mismo tiempo, también somos la solución a tan insólita destrucción, entendiendo claro está que debemos corregir, o más bien, cambiar por completo nuestra manera de pensar y de actuar sobre la naturaleza.

Por último y de acuerdo con Leff (2002):

La construcción de una racionalidad ambiental implica la formación de un nuevo saber y la integración interdisciplinaria del conocimiento, para explicar el comportamiento de sistemas socioambientales complejos. El saber ambiental problematiza el conocimiento fraccionado en disciplinas y la administración sectorial del desarrollo, para constituir un campo de conocimientos teóricos y prácticos orientado hacia la rearticulación de las relaciones sociedad-naturaleza (p. 227).

\section{Referencias bibliográficas}

Alea, A. (9 de mayo de 2007). Historia de la Educación Ambiental. Recuperado de: www.revistafuturos.info

Andreu, N. y Hernández, J.E. (1999). Importancia de la interdisciplinariedad para una cultura geográfica a favor de la educación. Educación, 98, 9-16.

Carabias, V. (2002). Conciencia ambiental y comportamiento ecológico. Revista internacional de sociología, 33, 133-170.

Corraliza, J.A. (2001). El comportamiento humano y los problemas ambientales. Estudios de psicología-Psicología ambiental 22(1), 3-10. Covas Álvarez, Onelia. Educación ambiental a partir de tres enfoques: comunitario, sistémico e interdisciplinario. Revista lberoamericana de Educación. 2004. Pág. 1

González, F. (1999). Producción de conocimiento e identificación de la problemática ambiental, en relación al horizonte dado por el uso de los conceptos de modernidad y posmodernidad. En Ambiente y Desarrollo Revista de educación y cultura. № 43, marzo (1999. pp. 21-53). Pontificia Universidad Javeriana. Bogotá, D.C.: IDEADE.
González, M.C. (1999). Principales tendencias y modelos de la Educación Ambiental en el sistema escolar. Revista Iberoamericana de Educación, $\mathrm{N}^{\circ} 11$.

Ibarra, G. (1999). Escenarios de la educación ambiental en la educación superior. Educación y Ciencia 2(1), 65-78.

Leff, E. (2002). Saber ambiental: sustentabilidad, racionalidad, complejidad, poder. México: Siglo XXI Editores.

Londoño, J.P. (2004). El desarrollo del pensamiento ambiental moderno. Gestión y ambiente 7(1), 49-58.

López, R. (1999). La investigación de problemas ambientales orientada a la resolución de los mismos, como un posible modelo de incorporación de la educación ambiental en el currículo. Innovación Educativa, 9, 269-280.

Mahecha, C. G. (2003). La educación ambiental: ¿solución a un problema o un problema por resolver? Ambiente y Desarrollo, № 12 junio. pág. 12, 81-95.

Navarro, J. (1997). La educación ambiental: una visión sintética de los problemas ambientales y su relación con otras esferas del quehacer humano. Revista Tarby, $17,53-61$.

Novo, M. (1996). La educación ambiental formal y no formal: dos sistemas complementarios. Revista Iberoamericana de Educación, 11, 75-102.

Rengifo R, B. (2012). et al. La educación ambiental una estrategia pedagógica que contribuye a la solución de la problemática ambiental en Colombia. XII coloquio internacional de geocrítica. pág. 4, 1-16

Ruvalcaba, J.C. (2002). Análisis de la práctica docente en la enseñanza de la Ecología. Revista de la Universidad del Valle de Atemajac 16(43), 83-87.

Barcelona, M. (2008). et al. Secretaría de ambiente. Ministerio de educación. Secretaría de educación. Gobierno de la provincia de Córdoba. Pág. 3.

Terrón Amigón, Esperanza. La educación ambiental ante los desafíos del siglo XXI. Revista de la academia Mexicana de profesores de Ciencias naturales. № 3. Enero diciembre. Pág. 5 - 13. 2000

Valdés, O. (2001) ¿Cómo la educación ambiental contribuye a proteger el medio ambiente?: concepción, estrategias, resultados y proyecciones en Cuba. Ministerio de educación. 2001 